OPEN ACCESS

Edited by:

Ghassan M. Matar,

American University of Beirut,

Lebanon

Reviewed by:

Sylvie Baucheron,

Institut National de la Recherche

Agronomique, France

Miklos Fuzi,

Semmelweis University, Hungary

*Correspondence:

Sinisa Vidovic

svidovic@umn.edu

Specialty section:

This article was submitted to

Antimicrobials, Resistance

and Chemotherapy,

a section of the journal

Frontiers in Microbiology

Received: 31 December 2018

Accepted: 25 March 2019

Published: 09 April 2019

Citation:

Vidovic S, An R and Rendahl A (2019) Molecular and Physiological

Characterization

of Fluoroquinolone-Highly Resistant

Salmonella Enteritidis Strains.

Front. Microbiol. 10:729.

doi: 10.3389/fmicb.2019.00729

\section{Molecular and Physiological Characterization of Fluoroquinolone-Highly Resistant Salmonella Enteritidis Strains}

\author{
Sinisa Vidovic*, Ran An and Aaron Rendahl \\ Department of Veterinary and Biomedical Sciences, University of Minnesota, Saint Paul, MN, United States
}

Four clinical isolates of Salmonella Enteritidis, susceptible to ciprofloxacin, and their spontaneous ciprofloxacin resistant (MICs from 8 to $16 \mu \mathrm{g} / \mathrm{mL}$ ) and highly resistant (MIC $2048 \mu \mathrm{g} / \mathrm{mL}$ ) mutants were used to gain an insight into the dynamics of development of fluoroquinolone (FQs) resistance in S. Enteritidis serovar. The first two high-frequency (i.e., mutations that occurred in each tested strain) mutations occurred in the gyrA, resulting in amino acid substitutions S83Y and S83F as well as D87G. Amino acid substitution D87G was significantly associated with the highly resistant mutants. Another high-frequency mutation, deletion in the ramRA intergenic region, was determined among the same group of highly resistant mutants. More importantly, each of these deletion mutations affected the RamR binding site. The effect of one $41 \mathrm{bp}$ deletion mutation was empirically tested. The results showed that the deletion was responsible for resistance to ceftiofur and amoxicillin/clavulanic acid and decreased susceptibility to azithromycin and tetracycline. Performing gene expression assays across all ciprofloxacin susceptible groups, we found a consistent and significant upregulation of the $\operatorname{ram} A, \operatorname{acr} B$, and to/C (efflux pump associated genes) and downregulation of $\mathrm{ompF}$ (porin), clearly illustrating the importance of not only efflux but also porin-mediated permeability in the development of FQs resistance. Our data also showed that $S$. Enteritidis could acquire multiple mutations in QRDR region, further resulting in no up regulation of the ram $A, a c r B$ and tolC genes. These QRDR mutations and no activation of the AcrAB efflux pump seem to preserve the fitness of this organism compared to the $S$. Enteritidis strains that did not acquire multiple QRDR mutations. This report describes the dynamics of FQ-associated mutations in the highly resistant in FQ mutants in $S$. Enteritidis. In addition, we characterized a deletion in the ramRA integenic region, demonstrating that this frequent mutation in the highly resistant $F Q$ mutants provide resistance or reduce susceptibility to multiple families of antibiotics.

Keywords: Salmonella, quinolone resistance, multidrug resistance, AcrAB efflux pump and small-colony variant phenotype, OmpF porin 


\section{INTRODUCTION}

Non-typhoidal Salmonella (NTS) is a major zoonotic pathogen worldwide (Bangtrakulnonth et al., 2004; Scallan et al., 2011). Infections caused by this pathogen have been mainly associated with gastroenteritis, an acute self-limiting intestinal infection. However, it has been shown that in different regions of the world, especially in places with high percentages of immunocompromised populations, NTS is a frequent cause of bacteremia (Graham, 2010; Reddy et al., 2010; Okoro et al., 2012), an invasive life-threatening extra intestinal infection. Once acquired, this invasive salmonellosis may result in a fatality rate of $20 \%$ (Gordon, 2008). Multidrug resistance and in particular resistance to fluoroquinolones (FQs), a potent broad-spectrum family of antibiotics used for the primary treatment of invasive salmonellosis, play a key role in the treatment failure (Pers et al., 1996; Rupali et al., 2004).

Fluoroquinolones resistance in NTS can be acquired through transmissible quinolone-resistance mechanisms (MartinezMartinez et al., 1998; Redgrave et al., 2014; Hooper and Jacoby, 2015). Transmissible quinolone-resistance occurs via the horizontal transfer of plasmids, which carry a family of $q n r$ genes (i.e., $q n r A$, $q n r B$, $q n r S, q n r C$, and $q n r D)$-also known as plasmid-mediated quinolone resistance (PMQR) genes. It has been shown that the PMQR genes confer modest resistance against FQs (Martinez-Martinez et al., 1998). Salmonella spp., most commonly vertically acquired resistance to FQs through de novo mutations, which occurs in the quinolone resistance-determining region (QRDR) of the $g y r A$ and parC genes (Piddock et al., 1998; O’Regan et al., 2009; Ricci and Piddock, 2009) and in genes encoding the acrAB-tolC efflux system (Baucheron et al., 2004) as well as in genes that encode regulators of the efflux system (Abouzeed et al., 2008; O'Regan et al., 2009; Blair et al., 2015). Vertically acquired mutations play a critical role in antimicrobial treatments, as these mutations occur quickly under the selective pressure of the drug, resulting in a high level of resistance against FQs, which may subsequently lead to antimicrobial treatment failure. The vertical evolution of resistance-conferring mutations is very complex. The outcome, antimicrobial susceptibility, depends on a sum of the interactions between antagonistic and synergistic resistance-conferring mutations rather than on an independent effect of a single mutation (Hartl, 2014). Understanding the development of de novo mutations, their interactions, and physiological adaptation of the bacterial organism to the selective pressure imposed by the drug is critically important in antimicrobial stewardship programs.

In this study, we examined the development of the resistance and the high-level resistance to FQs using four ciprofloxacin susceptible Salmonella enterica serovar Enteritidis (S. Enteritidis) isolates and their spontaneous ciprofloxacin resistant (MIC ranging from 8 to $16 \mu \mathrm{g} / \mathrm{mL}$ ) and highly resistant (MIC $2048 \mu \mathrm{g} / \mathrm{mL}$ ) mutants. The aim of this study was to determine the type and dynamics of the mutations and the overall physiological adaptations associated with the development of extremely high resistance to ciprofloxacin.

\section{MATERIALS AND METHODS}

\section{Strains of S. Enteritidis}

Out of $88 \mathrm{~S}$. Enteritidis strains, implicated in human and avian infections, we selected four strains that exhibited the most profound susceptibility to ciprofloxacin. The following four $S$. Enteritidis strains: A-5 from Texas (MIC $0.015 \mu \mathrm{g} / \mathrm{mL}$ ), A-7 from Pennsylvania (MIC $0.06 \mu \mathrm{g} / \mathrm{mL}$ ), A-21 from Iowa (MIC $0.03 \mu \mathrm{g} / \mathrm{mL}$ ), and A-33 from Wisconsin (MIC $<0.0009375 \mu \mathrm{g} / \mathrm{mL})$ were selected for the study. All four strains were implicated in the primary infections of avian hosts. The strains were received from the National Veterinary Services Laboratories (NVSL), Ames, IA. The $S$. Enteritidis strains were diagnosed at the NVSL using standard microbiological and serological methods for Salmonella identification and classification. The strains were checked for their purity upon their arrival by plating them on Luria-Bertani (LB) agar (Difco) plates, followed by an overnight incubation at $37^{\circ} \mathrm{C}$. After confirmation of the strains' purity, they were stored at $-80^{\circ} \mathrm{C}$ in LB broth (Difco) with 10\% glycerol.

\section{Antimicrobial Susceptibility Tests}

Four S. Enteritidis strains, A-5, A-7, A-21, and A-33, were examined for their antimicrobial susceptibilities using Sensititre CMV3AGNF plates (TREK Diagnostic Systems, Cleveland, $\mathrm{OH}$, United States). The Sensititre plates each contained 14 antimicrobial agents dosed in 96 wells at appropriate dilutions, as specified by NARMS (National Antimicrobial Resistance Monitoring System) of the CDC. Each well of the Sensititre microtiter plate was inoculated according to the instructions of the manufacturer, followed by incubation at $37^{\circ} \mathrm{C}$ for $18-22 \mathrm{~h}$. The minimal inhibitory concentration (MIC) breakpoints were determined according to the National Committee for Clinical Laboratory Standards (NCCLS) M07-A10 (Clinical and Laboratory Standards Institute [CLSI], 2015a) and M100-S25 (Clinical and Laboratory Standards Institute [CLSI], 2015b).

\section{Induction of FQ Resistance and Selection for Resistant and Highly Resistant Mutants}

To obtain this collection, we exposed each parental strain at its mid-exponential growth phase to $0.02 \mu \mathrm{g} / \mathrm{mL}$ of ciprofloxacin, followed by an overnight incubation period. After the overnight incubation, ciprofloxacin-challenged cultures were inoculated (1:100) into LB broth, containing a twofold higher concentration of ciprofloxacin compared to the previous ciprofloxacin challenge. Further, a series of twofold increase ciprofloxacin concentrations (from 0.04 to $2.4 \mu \mathrm{g} / \mathrm{mL}$ ) challenges were undertaken, and a selection of the ciprofloxacin resistant mutants took place on LB agar plates with $4 \mu \mathrm{g} / \mathrm{mL}$ of drug concentration. Similarly, after a series of twofold increase ciprofloxacin concentrations (from 8 to $32 \mu \mathrm{g} / \mathrm{mL}$ ) the highly resistant strains were selected on LB agar plates, which contained $40 \mu \mathrm{g} / \mathrm{mL}$ of ciprofloxacin. 


\section{Minimal Inhibitory Concentration (MIC) Determinations}

The MICs of ciprofloxacin for the parental strains and their resistant and highly resistant spontaneous mutants were determined using broth macrodilution method, as described by the NCCLS M07-A10 (Clinical and Laboratory Standards Institute [CLSI], 2015a) and M100-S25 (Clinical and Laboratory Standards Institute [CLSI], 2015b).

\section{Cross-Resistance Assay}

After selecting the resistant, A-5 (R), A-7 (R), A-21 (R), and A-33 (R) and the highly resistant mutants, A-5 (HR), A-7 (HR), A-21 (HR), and A-33 (HR), they were examined for any additional acquired antimicrobial resistance phenotype, which is not relevant to FQ class of antibiotics. The susceptibility of the resistant and the highly resistant strains was determined using Sensititre CMV3AGNF plates, as described above.

\section{gyrAB, parCE, acrAB-tolC, ramAR, rpoE, $c p \times R$, ompFC, and IpXA Full-Length Genes Sequencing and Single Nucleotide Polymorphisms (SNPs) Analysis}

The list of the primers used for the full-length gene sequencing of gyrA, gyrB, parC, parE, acrA, acrB, tolC, ramA, ramR, rpoE, $c p x R, o m p F, o m p C$, and $l p x A$ is shown in Table 1. The primers for $a c r A, a c r B, \operatorname{tol} C, \operatorname{ram} A, \operatorname{ram} R, r p o E$, and $c p x R$ are designed to target the promoter regions of these genes as well. The amplicons were generated by Platinum Taq DNA polymerase (Thermo Fisher Scientific) and were prepared for DNA sequencing by the Prism BigDye Terminator cycle sequencing kit (Applied Biosystems, Foster City, CA, United States). The nucleotide sequences on both strands were determined using an ABI 3730 x 1 DNA analyzer (Genomics Center, University of Minnesota, Minneapolis, MN, United States). Each strand was checked and then aligned with its complementary strand. A consensus DNA sequence was obtained using Clustal Omega (Larkin et al., 2007). The annotated DNA sequences were exported into Molecular Evolutionary Genetics Analysis (MEGA) version 7 (Tamura et al., 2007) for the identification of non-synonymous single nucleotide polymorphisms (nsSNPs) within the coding regions and SNPs within the promoter regions of $\operatorname{acr} A, \operatorname{acr} B, \operatorname{tol} C, \operatorname{ram} A, \operatorname{ram} R$, $r p o E$, and $c p x R$. Nucleotide sequence translation was carried out using EMBOSS Transeq (Kearse et al., 2012) (the European Molecular Biology Laboratory-European Bioinformatics Institute; Hinxton, Cambridge, United Kingdom).

\section{Gene Expression Assay}

Overnight cultures of the susceptible, resistant, and highly resistant $S$. Enteritidis A-5, A-7, A-21, and A-33 strains were diluted $1 / 100$ in $100 \mathrm{~mL}$ of $\mathrm{LB}$ and grown at $37^{\circ} \mathrm{C}$ with constant shaking at $190 \mathrm{rpm}$ to optical density at $600 \mathrm{~nm}$ of 0.5 . These mid-exponential growth phase cultures were exposed to ciprofloxacin at a final concentration of $0.010 \mu \mathrm{g} / \mathrm{mL}$. The cultures were additionally incubated for $30 \mathrm{~min}$ and harvested by centrifugation. The total RNAs were extracted using the RNeasy Mini kit (Qiagen), following the manufacturer's instructions. The synthesis of complementary DNA (cDNA) was carried out using iScriptTM Reverse Transcription (BioRad Laboratories, Inc. Hercules, CA, United States). Quantitative PCR was performed using Power SYBR green master mix kit (Applied Biosystems). The primers used to detect transcripts of $\operatorname{ram} A, \operatorname{ram} R$, acrA, tolC, rpoE, cpxR, ompA, ompW, ompF, $o m p C$, and $s l y B$ are listed in Supplementary Table S1. The gapA gene was selected as an internal reference control and the data were reported as the fold change relative to the levels in the susceptible strains using the comparative $C_{T}$ method (Schmittgen and Livak, 2008).

\section{Complementation Assay}

The $\operatorname{ram} R A$ sequence, including its intergentic region, was amplified by PCR using genomic DNA of $S$. Enteritidis 5-A highly resistant strain, Q5 High-Fidelity DNA polymerase (New England BioLabs, Ipswich, MA, United States), and the primers ramRA F (5'-GCG GGA TCC GAC AGT GAT GTT CAG TGA AC-3') and ramRA R (5' - TCA GTC GAC CTC TTG CTC GGC GCG CTG GA-3'); the BamHl and SaIl sites are underlined. The $\operatorname{ramRA}$ fragment was double digested and cloned between the BamHl and SalI restriction sites of the low copy expression vector pTrc99A (Life Science Market). The recombinant pTrc99A vector was sequenced and an insertion of the $\operatorname{ram} R A$ haplotype of S. Enteritidis 5-A highly resistant strain was confirmed. The $S$. Enteritidis 5-A susceptible strain was transformed with the recombinant $\mathrm{p} \operatorname{Trc} 99 \mathrm{~A}$ vector and also with an empty pTrc99A vector, respectively. Both $S$. Enteritidis A-5 susceptible strains, complemented with the recombinant and non-recombinant pTrc99A vectors, were tested for susceptibility to the panel of 10 antimicrobial agents, as described above.

\section{Growth Curve Assay}

The difference in the growth kinetics between the parental ciprofloxacin susceptible strain and its spontaneous highly resistant mutant with a small colony variants (SCVs) phenotype was determined by measuring biomass at $600 \mathrm{~nm}$. The overnight cultures of the parental and highly resistant mutant strains were diluted and normalized to an optical density equivalent to 0.5 McFarland standard $\left(1.5 \times 10^{8} \mathrm{CFU} / \mathrm{mL}\right)$. These normalized cultures were used to inoculate $50 \mathrm{~mL}$ of freshly prepared LB, followed by incubation at $37^{\circ} \mathrm{C}$ with shaking at $180 \mathrm{rpm}$. The $\mathrm{OD}_{600}$ values were measured every $60 \mathrm{~min}$ for $6 \mathrm{~h}$. In addition, the differences in the growth kinetics between the parental strains A-5, A-7, A-21, and A-33 and their resistant mutants were measured. This growth assay was carried out with addition of $0.005 \mu \mathrm{g} / \mathrm{mL}$ of ciprofloxacin as described above.

\section{Statistical Analysis}

To test for differences in gene expression between resistant and highly resistant strains, compared with their parental susceptible strains, $t$-tests were performed for each strain/gene/resistance combination. To better estimate variability, variance was pooled for data from the same strain. Additionally, $p$-values were adjusted for multiple comparisons using the Bonferroni-Holm correction, again, within each strain separately. All tests were performed on the ddCt scale, and results transformed to fold 
TABLE 1 | Primers used for the amplification and full gene sequencing

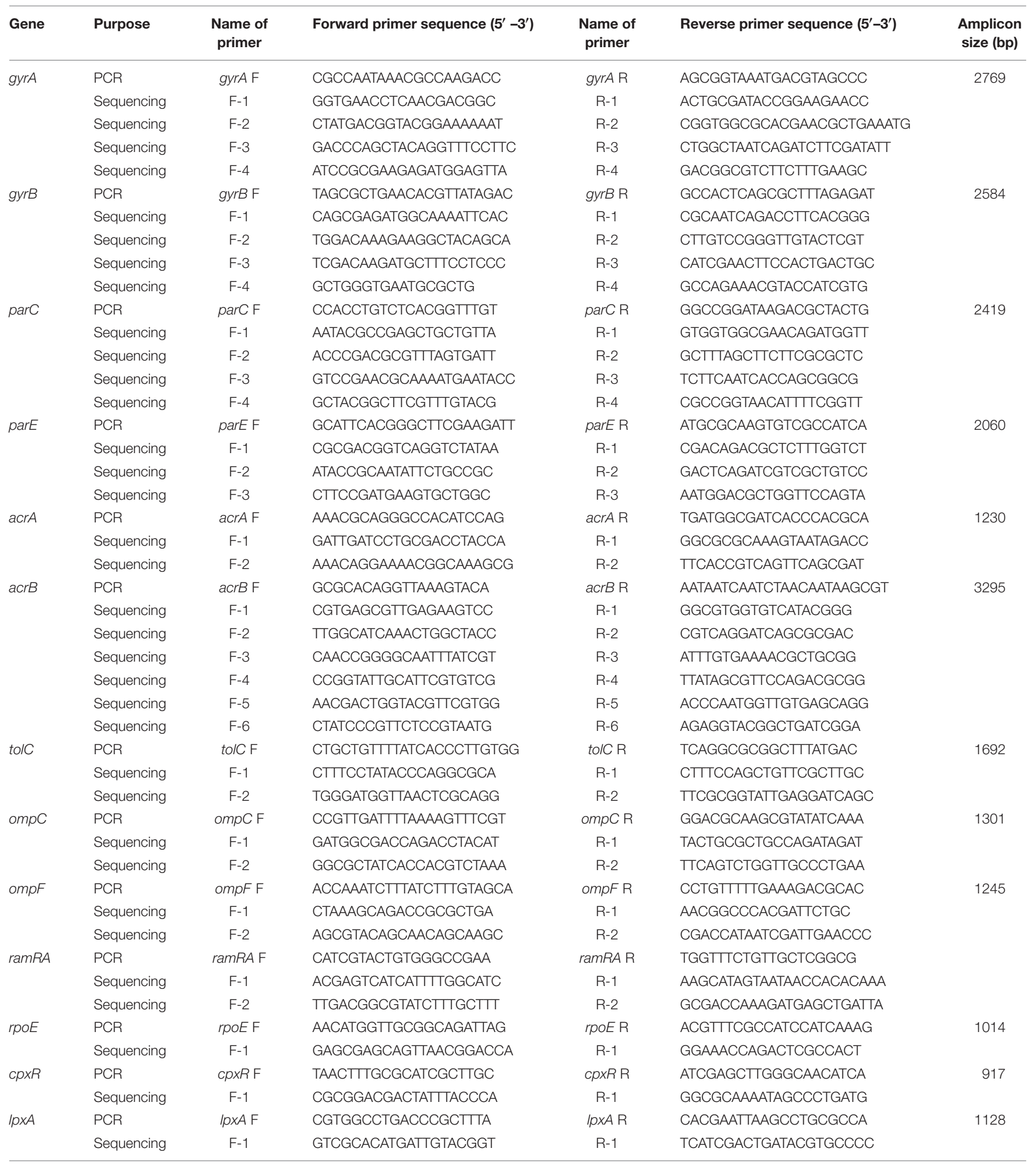

change for presentation. Growth curves kinetics were analyzed by CoStat version 6.4 software (Co-Hort Software, Monterey, CA, United States) using homogeneity of linear regression slopes method to test for significant $(p<0.05)$ differences.

\section{Nucleotide Sequence}

\section{Accession Numbers}

Nucleotide sequences of each gene haplotype were deposited in GenBank under accession numbers MH933946 to MH933963. 
Also, accession numbers for six ramAR haplotypes, including their intergenic regions, range from MK024405 to MK024410.

\section{RESULTS}

\section{Selection and Characterization of Spontaneous Ciprofloxacin-Resistant and Highly Resistant $S$. Enteritidis Mutants}

Four clinical strains of $S$. Enteritidis with a high level of ciprofloxacin susceptibility, A-5 (MIC $0.015 \mu \mathrm{g} / \mathrm{mL}$ ), A7 (MIC $0.06 \mu \mathrm{g} / \mathrm{mL}$ ), A-21 (MIC $0.03 \mu \mathrm{g} / \mathrm{mL}$ ), and A-33 $(\mathrm{MIC}<0.0009 \mu \mathrm{g} / \mathrm{mL})$, were used to create a collection of spontaneous ciprofloxacin resistant and highly resistant mutants. Following the selection of the ciprofloxacin resistant and highly resistant mutants, we tested these two groups of mutants for their MIC values to ciprofloxacin. The resistant mutant strains exhibited MIC values ranging from 8 to $16 \mu \mathrm{g} / \mathrm{mL}$, while their highly resistant counterparts achieved an MIC of $2048 \mu \mathrm{g} / \mathrm{mL}$, indicating much higher MIC values compared to those of the resistant mutant strains (Table 2).

\section{Exposure of S. Enteritidis to Ciprofloxacin Leads to the Development of Cross-Resistance}

To determine the potential side effect of the ciprofloxacin treatment on antimicrobial susceptibility of the spontaneous mutants to antibiotics that are not related to the family of FQs, we carried out the antimicrobial susceptibility tests for the parental strains and their spontaneous resistant and highly

TABLE 2 | Summary of MICs, amino acid substitutions, deletion mutations in the ramRA intergenic region and gene expression fold changes for the ramA, acr $B$ and $O \mathrm{mpF}$.

\begin{tabular}{|c|c|c|c|c|c|c|c|}
\hline & \multirow{2}{*}{$\frac{\text { MIC(ug/mL) }}{\text { Ciprofloxacin }}$} & \multicolumn{2}{|c|}{$\begin{array}{l}\text { Amino acid } \\
\text { substitutions }\end{array}$} & \multirow{2}{*}{$\begin{array}{c}\begin{array}{c}\text { Deletion } \\
\text { mutations }\end{array} \\
\text { RamRA }\end{array}$} & \multicolumn{3}{|c|}{$\begin{array}{l}\text { Gene expression } \\
\text { fold change }\end{array}$} \\
\hline & & GyrA & ParC & & $\operatorname{ramA}$ & $a c r B$ & ompF \\
\hline A5-S & 0.015 & & & & 1 & 1 & 1 \\
\hline A5-R & 16 & $\begin{array}{l}\text { S83Y; } \\
\text { D87G }\end{array}$ & S80R & & 1.3 & 2.7 & -2.9 \\
\hline A-5-HR & 2048 & $\begin{array}{l}\text { S83Y; } \\
\text { D87G }\end{array}$ & S80I & $\begin{array}{l}41 \mathrm{nt}+1 \\
\text { mutation }\end{array}$ & 80 & 6.6 & -7.7 \\
\hline A-7-S & 0.06 & & & & 1 & 1 & 1 \\
\hline$A-7-R$ & 16 & S83Y & & & 11 & 3.6 & -25 \\
\hline A-7-HR & 2048 & $\begin{array}{l}\text { S83Y; } \\
\text { D87G }\end{array}$ & G78D & $9 \mathrm{nt}$ & 83.3 & 10.2 & -3.8 \\
\hline A-21-S & 0.03 & & & & 1 & 1 & 1 \\
\hline$A-21-R$ & 8 & S83Y & & $11 \mathrm{nt}$ & 72.2 & 8.3 & -6.7 \\
\hline $\mathrm{A}-21-\mathrm{HR}$ & 2048 & $\begin{array}{l}\text { S83F; } \\
\text { D87G }\end{array}$ & & $\begin{array}{l}12 \mathrm{nt}+1 \\
\text { mutation }\end{array}$ & 205.8 & 14.5 & -16.7 \\
\hline A-33-S & $<0.0009$ & & & & 1 & 1 & 1 \\
\hline A-33-R & 16 & S83F & & & 35.8 & 5.6 & -100 \\
\hline A-33-HR & 2048 & $\begin{array}{l}\text { S83F; } \\
\text { D87G }\end{array}$ & & $4 \mathrm{nt}$ & 22.6 & 7.4 & -43.3 \\
\hline
\end{tabular}

resistant mutants using ten, non-FQs-related antibiotics. The susceptibility tests illustrated the existence of four distinct phenotypes associated with the antimicrobial susceptibility profiles of the parental and their spontaneous mutant strains (Table 3). The first phenotype included no change of MICs for amoxicillin/clavulanic acid and ampicillin between the parental strains and their mutants (Table 3). The second phenotype showed a slight increase of MICs for ceftriaxone and gentamycin between the parental and their mutant strains (Table 3). The third phenotype was characterized by a variable change of MICs for tetracycline and streptomycin among the four $S$. Enteritidis parental strains and their spontaneous mutants (Table 3). For instance, the parental A-5 strain and its resistant and highly resistant mutant strains had the same MICs values of $<4 \mu \mathrm{g} / \mathrm{mL}$ for tetracycline. In contrast to this group of A-5 strains, parental A-7 strain exhibited an MIC of $<4 \mu \mathrm{g} / \mathrm{mL}$; however, its resistant and highly resistant mutants acquired MICs of $8 \mu \mathrm{g} / \mathrm{mL}$ and $32 \mu \mathrm{g} / \mathrm{mL}$, respectively, for the same antibiotic. The fourth phenotype was characterized by an increase in the MICs between the parental strains and their resistant and highly resistant mutants (Table 3). The MICs values for amikacin, chloramphenicol, cefoxitin, azithromycin, and ceftiofur of all of the $S$. Enteritidis strains have been accurately correlated to their ciprofloxacin MICs. The highest MICs values for these five antibiotics demonstrated ciprofloxacin highly resistant strains, whereas the lowest MICs values for the same five antibiotics exhibited the ciprofloxacin susceptible (i.e., parental) strains.

\section{Mutations Associated With the Ciprofloxacin Resistant and Highly Resistant Strains}

To identify mutations that contribute to the development of FQsresistance (MIC from 8 to $16 \mu \mathrm{g} / \mathrm{mL}$ ) and high resistance (MIC $2048 \mu \mathrm{g} / \mathrm{mL}$ ), we sequenced the genes that encode topoisomerase II (gyrA, gyrB, parC, parE), efflux pump ( $\operatorname{cr} A$, acrB, tolC), efflux pump regulators $(\operatorname{ram} R, \operatorname{ram} A)$, porins $(o m p F, o m p C)$, extracytoplasmic stress response regulators ( $r p o E, c p x R)$, and liposaccharide component $(p x A)$ of the four parental strains along with their resistant and highly resistant mutants. No mutation was detected in the coding sequences of $g y r B$, parE, acr $A, a c r B$, tolC, ram $A, r a m R, o m p F, o m p C, r p o E, c p x R$, and $l p x A$. In addition, the entire promoter regions of the $\operatorname{acr} A, \operatorname{acr} B, \operatorname{tol} C$, $r p o E$, and $c p x R$ genes were intact, with no introduced mutations in either ciprofloxacin-resistant or ciprofloxacin-highly resistant mutants. A single non-synonymous substitution, G78D, was present in the parC of the highly resistant A-7 mutant strain. Another two amino acid substitutions, S80R and S80I, were present in the same gene of the resistant A-5 and highly resistant A-5 strains, respectively. In addition to these three amino acid substitutions, no other mutation was detected in the parC of the resistant A-7, resistant A-21, highly resistant A-21, resistant A33 , and highly resistant A-33 mutant strains. In contrast to the sporadic occurrence of mutations in parC, the gyrA was found to be a common target for the development of FQs resistance in the tested $S$. Enteritidis strains (Figure 1A and Table 2). The two FQs-resistant mutants, A-7 and A-21, acquired the same 
TABLE 3 | Antimicrobial susceptibilities profiles of four parental S. Enteritidis strains and their resistant and highly resistant ciprofloxacin mutants.

\begin{tabular}{|c|c|c|c|c|c|c|c|c|c|c|c|c|}
\hline \multicolumn{13}{|c|}{ FQ Susceptibility groups } \\
\hline \multirow[t]{2}{*}{ Strain IDs } & \multicolumn{4}{|c|}{ Susceptible } & \multicolumn{4}{|c|}{ Resistant } & \multicolumn{4}{|c|}{ Highly resistant } \\
\hline & A-5 & $A-7$ & $A-21$ & A-33 & A-5 & A-7 & $A-21$ & A-33 & A-5 & A-7 & A-21 & A-33 \\
\hline \multicolumn{13}{|l|}{ No change of MIC ( $\mu \mathrm{g} / \mathrm{mL})$} \\
\hline Amoxicillin/ Clavulanic acid & $<1 / 0.5$ & $<1 / 0.5$ & $<1 / 0.5$ & $<1 / 0.5$ & $<1 / 0.5$ & $<1 / 0.5$ & $<1 / 0.5$ & $<1 / 0.5$ & $<1 / 0.5$ & $<1 / 0.5$ & $<1 / 0.5$ & $<1 / 0.5$ \\
\hline Ampicillin & $<1$ & $<1$ & $<1$ & $<1$ & $<1$ & $<1$ & $<1$ & $<1$ & $<1$ & $<1$ & $<1$ & $<1$ \\
\hline \multicolumn{13}{|l|}{ Slight change of MIC } \\
\hline Ceftriaxone & $<0.25$ & $<0.25$ & $<0.25$ & $<0.25$ & 0.5 & 1 & 0.5 & 1 & 0.5 & 1 & 1 & 1 \\
\hline Gentamicin & 2 & 2 & 1 & 4 & 4 & 2 & 1 & 2 & 4 & 1 & 4 & 2 \\
\hline \multicolumn{13}{|l|}{ Variable change of MIC } \\
\hline Tetracycline & $<4$ & $<4$ & $<4$ & $<4$ & $<4$ & 8 & $<4$ & 8 & $<4$ & 32 & 8 & $<4$ \\
\hline Streptomycin & 64 & 64 & 64 & $>64$ & $>64$ & $>64$ & 64 & $>64$ & $>64$ & $>64$ & 64 & $>64$ \\
\hline \multicolumn{13}{|c|}{ Increase of MIC (Cross-resistance) } \\
\hline Amikacin & 8 & 32 & 8 & 16 & 32 & $>64$ & $>64$ & $>64$ & $>64$ & $>64$ & $>64$ & $>64$ \\
\hline Azithromycin & 0.12 & 0.25 & 0.12 & 0.25 & 4 & 8 & 8 & 8 & 8 & $>16$ & $>16$ & $>16$ \\
\hline Chloramphenicol & 4 & 8 & 4 & 4 & 8 & 32 & 16 & 32 & 32 & $>32$ & $>32$ & 32 \\
\hline Cefoxitin & 2 & 4 & 1 & 2 & 8 & $>32$ & $>32$ & $>32$ & 32 & $>32$ & $>32$ & $>32$ \\
\hline Ceftiofur & 1 & 1 & 1 & 1 & 2 & 8 & 4 & 8 & 4 & 8 & 8 & 8 \\
\hline
\end{tabular}

amino acid substitution, S83Y, the resistant A-33 mutant received another amino acid substitution, S83F, while the resistant A5 mutant obtained two amino acid substitutions, S83Y and D87G (Table 2). The highly resistant mutants acquired a highfrequency mutation, the second amino acid substitutions, D87G (Table 2). The second high-frequency mutation associated with an extremely high level of ciprofloxacin resistance was identified in an intergenic region of $\operatorname{ramRA}$ (Figure 1B). This mutation was first identified among the resistant mutants, a single A-21 strain showed an 11-nt deletion (from 150 to 161 bp upstream of the $\operatorname{ram} A$ ), which corresponds to the RamR binding site (Figure 1C and Table 2). This mutation progressed as the drug concentration increased and reached its high frequency among the highly resistant mutant strains. All the highly resistant mutant strains acquired a deletion that disrupted the RamR binding site (Figure 1C). To further investigate the effect of the $\operatorname{ram} R A$ intergenic deletion, the $\operatorname{ramRA}$ haplotype of the highly resistant A-5 strain was cloned into pTrc99-A and transformed into the parental A-5 strain. The complemented strain exhibited resistance to ceftiofur, amoxicillin/clavulanic acid and decreased susceptibility to azithromycin, tetracycline and ciprofloxacin, compared to the same strain transformed with the empty vector (Table 4). It is interesting that the A-5 parental strain complemented with the pTrc99A::ramRA vector could not develop a full ciprofloxacin resistance but only a decreased susceptibility to this antibiotic.

\section{Gene Expression Analysis of the acrAB-to/C Efflux Pump, Its Regulators ramRA, Outer Membrane Components and Extracytoplasmic Regulators}

To compare the effect of the ciprofloxacin treatment on the gene expressions of the parental, resistant, and highly resistant strains, a subinhibitory concentration of this antibiotic for the A-5 parental strain was used. The selected ciprofloxacin concentration did not have any effect on the growth rate of this strain (data not shown). The expressions of the efflux pump genes, acr $A$, acr $B$, tolC, efflux pump regulators, $\operatorname{ram} R, \operatorname{ram} A$, extracytoplasmic regulators, $r p o E, c p x R$, and genes encoding porins, ompA, ompW, ompF, ompC, slyB for the parental strains and their resistant and highly resistant mutants are shown in Figure 2. Most notably, the expression of the $\operatorname{ramA}$ gene significantly increased in the all-highly resistant and resistant mutants except for the A-5 resistant mutant (Figure 2). The upregulation of the $\operatorname{ram} A$ gene ranged from 205 folds in the highly resistant A-21 strain to 10 folds in the resistant A-7 strain. Both genes associated with the acr $A B$-tol $C$ efflux pump, acr $B$ and tolC, showed significant $(p<0.05)$ levels of expression across the highly resistant and resistant strains. In general, the $\operatorname{acr} B$ exhibited a higher level of upregulation compared to that of the tolC gene. The highly resistant strains exhibited a higher expression of genes, $a c r B$ and tolC, compared to their resistant counterparts (Figure 2). In contrast to the efflux pump, gene encoding outer membrane porin $\mathrm{F}$, ompF, showed significant $(p<0.05)$ downregulation across the all-highly resistant and resistant mutant strains (Figure 2). Another gene-encoding outer membrane protein $\mathrm{C}$, ompC, was downregulated in all the resistant and highly resistant mutants but at a less significant rate compared to that of the $\operatorname{ompF}$ (Figure 2). The other five genes, $o m p A, o m p W, \operatorname{sly} B, c p x R$, and $r p o E$, displayed a strain dependent pattern of expression (Figure 2).

\section{Bacterial Growth Assay}

Exposure to a high concentration of ciprofloxacin (e.g., $40 \mu \mathrm{g} / \mathrm{mL}$ ) resulted in the acquisition of the SCV phenotype among the highly resistant A-7, A-21, and A-33 mutants (Figures 3A,B). To characterize the growth kinetics of the SCV 
A

\section{A5 - Susceptible \\ A7-Susceptible \\ A21 - Susceptible \\ A33 - Susceptible}

A5 - Resistant

A7- Resistant

A21 - Resistant

A33 - Resistant

A5 - Highly Resistant

A7 - Highly Resistant

A21 - Highly Resistant

A33 - Highly Resistant

\section{$\begin{array}{cc}83 & 87 \\ 1 & 1\end{array}$}

ARVVGDVIGKYHPHGDSAVYDTIVRMAQPFSLRYMLVDGQGNFGSI ARVVGDVIGKYHPHGDSAVYDTIVRMAQPFSLRYMLVDGQGNFGSI ARVVGDVIGKYHPHGDSAVYDTIVRMAQPFSLRYMLVDGQGNFGSI ARVVGDVIGKYHPHGDSAVYDTIVRMAQPFSLRYMLVDGQGNFGSI

ARVVGDVIGKYHPHGDYAVYGTIIVRMAQPFSLRYMLVDGQGNFGSI ARVGGDVIGKYHPHGDYAVYDTIVRMAQPFSLRYMLVDGQGNFGSI ARVGDVIGKYHPHGDYAVYDTIVRMAQPFSLRYMLVDGQGNFGSI ARVGDVIGKYHPHGDFAVYDTIVRMAQPFSLRYMLVDGQGNFGSI

ARVGDVVIGKYHPHGDYAVYGTIVRMAQPFSLRYMLVDGQGNFGSI ARVVGDVIGKYHPHGDYAVYGTIVRMAQPFSLRYMLVDGQGNFGSI ARVVGDVIGKYHPHGDFAVYGTIVRMAQPFSLRYMLVDGQGNFGSI ARVVGDVIGKYHPHGDFAVYGTIVRMAQPFSLRYMLVDGQGNFGSI

B

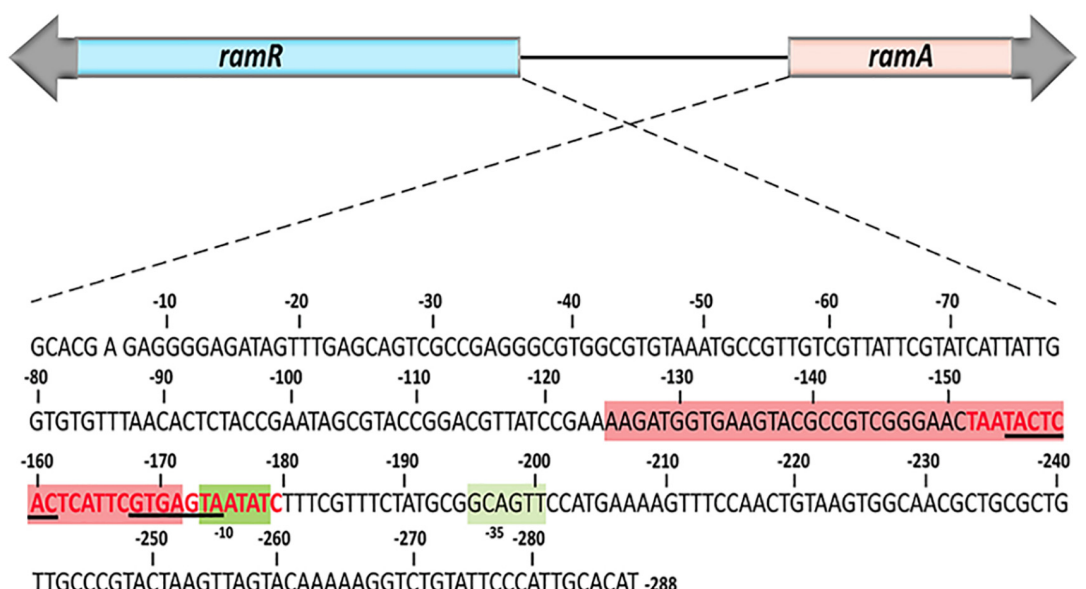

Deletion region associated with the over expression of the ramA gene NNN RamR binding site

Promoter sequence -10

Promoter sequence -35

_ Inverted repeat sequences

C

\begin{tabular}{|c|c|}
\hline & \\
\hline tible & TGGTGAAGTACGCCGTCGGGAACTAATACTCACTCATTCGTGAGTAATATC \\
\hline & CGGACGTTATCCGAAAAGATGGTGAAGTACGCCGTCGGGAACTAATACTCACTCATTCGTGAGTAATATC \\
\hline & CGGACGTTATCCGAAAAGATGGTGAAGTACGCCGTCGGGAACTAATACTCACTCATTCGTGAC \\
\hline & AAAAGATGGTGAAGTACGCCGTC \\
\hline
\end{tabular}

A5 - Resistant $\begin{array}{cccccccc}-110 & -120 & -130 & -140 & -150 & -160 & -170 & -180 \\ 1 & 1 & 1 & 1 & 1 & 1 & 1 & 1\end{array}$ A7 - Resistant CGGACGTTATCCGAAAAGATGGTGAAGTACGCCGTCGGGAACTAATACTCACTCATTCGTGAGTAATATCT CGGACGITATCCGAAAAGATGGTGAAGTACGCCGTCGGGAACTAATACTCACTCATTCGTGAGTAATATCT CGGACGTTATCCGAAAAGATGGTGAAGTACGCCGTCGGGAAC--------CATTCGTGAGTAATATCT

A21 - Resistant CGGACGTTATCCGAAAAGATGGTGAAGTACGCCGTCGGGAACTAATACTCACTCATTCGTGAGTAATATCT

A5 - Highly Resistant

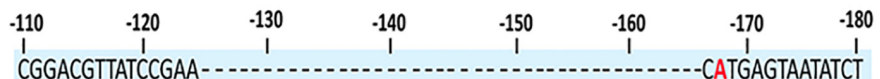
A7 - Highly Resistant

A21 - Highly Resistant GAAAGATGGTGAAGTACGCCGTCGGGACTAATACTCAC

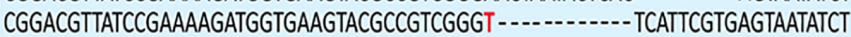

A33- Highly Resistant CGGACGTTATCCGAAAAGATGGTGAAGTACGCCGTCGGGAACTAATACTCACTC----GTGAGTAATATCT

FIGURE 1 | Continued 
FIGURE 1| The common mutations associated with the ciprofloxacin resistant and highly resistant mutants. (A) Comparative amino acid analysis of the GyrA identified amino acid substitutions S83Y and S83F among all four resistant mutants as well as double amino acid substitutions S83Y, S83F and D87G among all four highly resistant strains and one resistant strain. (B) Genetic organization of the ram locus, showing orientations of the ramR and ramA gens, their intergenic region with the RamR binding site, the ramA promoter sequences, inverted repeat sequences and deletion region associated with the highly resistant mutants. (C) Nucleotide sequence alignments of the ramRA intergenic regions of all three ciprofloxacin susceptible groups. Dash lines indicate deletion mutations, while the red color of nucleotide indicates nucleotide base substitution. The RamR binding site for $S$. enterica was determined by Baucheron et al. (2011).

and non-SCV mutants, we compared the growth curves of the A-21 and A-5 parental strains with their highly resistant mutants. Our data showed that the growth curve of the highly resistant A-21 mutant strain was significantly impaired $(p<0.05)$ compared to the growth curve of its parental strain (Figure 4), whereas the A-5 highly resistant mutant did not show significant growth alteration compared to its parental strain (Figure 4). To determine the effect of multiple QRDR mutations on $S$. Enteritidis fitness, we compared the growth curves of the A-5 resistant strain (i.e., GyrA S83Y, D87G; ParC S80R) and its A-5 parental strain (i.e., no QRDR mutations). There was no significant difference in the growth rate between these two strains (Figure 4). Next we compared the growth curves of the A-7 (GyrA S83Y), A-21 (GyrA S83Y) and A-33 (GyrA S83F) resistant strains with their parental strain (i.e., no QRDR mutations). The A-7, A-21 and A-33 resistant strains showed an impaired growth compared to those of other three strains (Figure 4).

\section{DISCUSSION}

Resistance to FQs class of antibiotics can be acquired vertically through de novo mutations and horizontally via the introduction of FQs resistance genes. The vertical evolution of FQs resistance can be very fast to allow the bacterial organism to develop resistance during a single antimicrobial treatment, which can lead to the treatment failure and death of a patient. Recently, Blair et al. (2015), examining the genomes of pre- and posttherapy isolates of $S$. Typhimurium from a patient who failed antimicrobial therapy, revealed a single mutation in the efflux pump gene, $\operatorname{acr} B$, which was acquired during the antimicrobial treatment. This novel G288D substitution in the drug-binding pocket of AcrAB-TolC multidrug efflux pump caused resistance

TABLE 4 | Differences in antimicrobial susceptibility of 5A susceptible strain and its counterparts complemented with an empty pTrc99A and pTrc99A::ramRA vectors.

\begin{tabular}{lccc}
\hline \multicolumn{3}{c}{ MIC $(\boldsymbol{\mu g} / \mathbf{m L})$} \\
\cline { 2 - 4 } Drug & $\begin{array}{c}\text { A-5 } \\
\text { Susceptible }\end{array}$ & $\begin{array}{c}\text { A-5 Susceptible } \\
\text { /pTrc99A }\end{array}$ & $\begin{array}{c}\text { A-5 Susceptible } \\
\text { /pTrc99A::ramRA }\end{array}$ \\
\hline Azithromycin & 0.12 & 0.25 & 8 \\
Amoxicillin/clavulanic & $<1 / 0.5$ & $8 / 4$ & $>32 / 16$ \\
acid & 1 & 1 & $>8$ \\
Ceftiofur & $<4$ & $<4$ & 32 \\
Tetracycline & 0.015 & 0.08 & 1 \\
Ciprofloxacin & & &
\end{tabular}

to the FQs group of antibiotics but susceptibility to the other drugs (e.g., doxorubicin and minocycline), underscoring the complexity and importance of vertical evolution in the development of antimicrobial resistance. Understanding the dynamics of drug-resistant mutations and their interactions and stress adaptive physiology of bacterial organism is important not only for rational drug design but also for predicting the evolution of antibiotic resistance in clinical settings. In the current study, using four ciprofloxacin susceptible $S$. Enteritidis clinical isolates in combination with stepwise selection, we determined the type and dynamics of the mutations associated with resistance and high-level resistance to ciprofloxacin as well as the stress adaptive response of the $S$. Enteritidis FQ mutant strains.

Among 14 genes, targeting topoisomerase II ( $g y r A$, gyrB, parC, parE), the AcrAB-TolC multidrug efflux pump ( $\operatorname{cr} A$, acr $B$, tolC), the efflux pump regulators $(\operatorname{ram} R, \operatorname{ram} A)$, porins $(o m p F, o m p C)$, extracytoplasmic stress response regulators $(r p o E, c p x R)$, and lipopolysaccharide component $(l p x A)$, the first high-frequency mutation in all the four resistant $S$. Enteritidis strains occurred in the QRDR of gyrA, resulting in S83Y and S83F substitutions. Among the population of the resistant strains, another amino acid substitution, S80R, in the parC was found only in the A5 strain, indicating the sporadic (i.e., mutation that occurred in one or two strains) nature of this mutation. As the resistance progressed, the second high-frequency amino acid substitution, D87G, was identified in the gyrA among the all-highly resistant strains. Among the group of the highly resistant strains, another sporadic amino acid substitution, G78D, occurred in the parC of the A-7 strain, confirming that the parC is not the primary target of ciprofloxacin resistance in $S$. Enteritidis.

It has been shown that, in $S$. Typhimurium, the regulator RamA plays a key role in the activation of AcrAB-TolC multidrug efflux pump (Abouzeed et al., 2008; Nikaido et al., 2008). O'Regan et al. (2009) showed that inactivation of AcrAB-TolC leads to an increased susceptibility to ciprofloxacin and other non-quinolone antibiotics, clearly indicating that the AcrABTolC efflux pump plays a role in fluroquinolone resistance and MDR in S. Enteritidis. Therefore, we examined the mutations in the coding and non-coding regions of the $\operatorname{ramRA}$ regulator in all-S. Enteritidis resistant and highly resistant mutant strains. The first mutation associated with the acr $A B$-tolC efflux pump was identified as an $11 \mathrm{bp}$ deletion in the $\operatorname{ramRA}$ intergenic region, affecting the RamR binding site. As the exposure of $S$. Enteritidis to drug concentration increased, deletions in the $\operatorname{ramRA}$ intergenic regions, affecting the RamR binding site, intensified, resulting in a maximum frequency among the group of highly resistant mutants. To determine the effect of this mutation on antimicrobial susceptibility of $S$. Enteritidis 

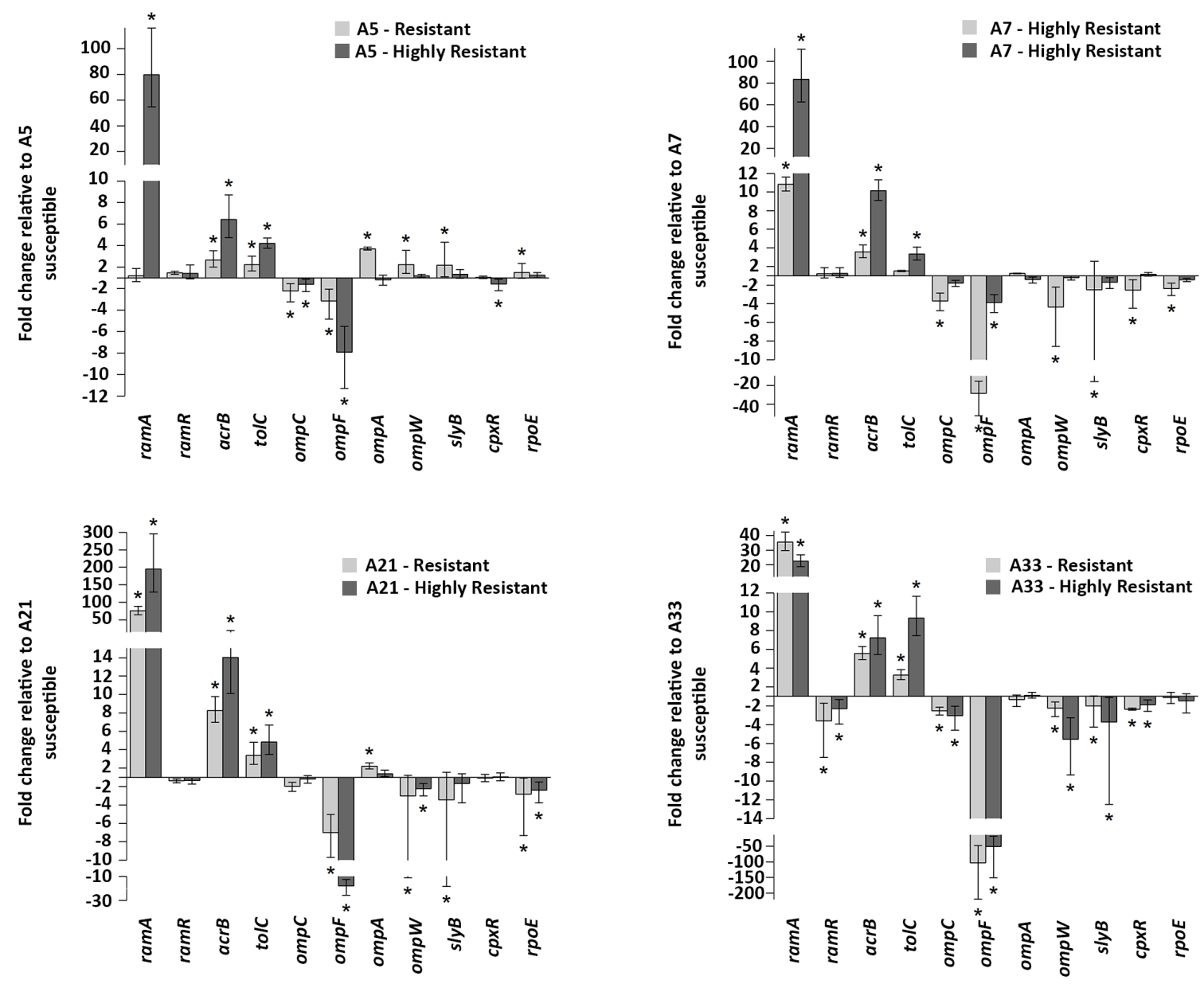

FIGURE 2 | Gene expressions. mRNA expression levels of acrB, tolC (efflux pump), ramR, ramA (regulators of the acrAB-tolC efflux pump), ompF, ompC, ompA, ompW, slyB (porins) and rpoE, cpxR (extracytoplasmic stress response regulators) in all ciprofloxacin susceptible groups. Values on the $y$ axis are relative expression levels (fold change) normalized against levels in the resistant and highly resistant strains. The data correspond to the mean values of three biological replications. Error bars correspond to the standard deviation. Asterisks indicate statistically significant differences $\left({ }^{*} p<0.05\right)$ in $t$-tests.

serovar, we complemented the A-5 parental strain with the recombinant plasmid, which contains the $\operatorname{ram} R A$ haplotype of its highly resistant A-5 mutant strain. The complemented parental strain showed complete resistance to ceftiofur and amoxicillin/clavulanic acid as well as decreased susceptibility to azithromycin and tetracycline, compared to its counterpart complemented with an empty plasmid. This experimental finding clearly points out that the deletion in the $\operatorname{ramRA}$ intergenic region, affecting the RamR binding site, plays a role in the development of multidrug resistance in $S$. Enteritidis. It is interesting that the complemented parental strain developed resistance against amoxicillin/clavulanic acid, whereas the highly resistant mutant exhibited susceptibility to the same drug. This discrepancy could be explained by the difference in the fitness landscape of these two organisms. Hartl (2014) showed that there are synergistic and antagonistic interactions among the resistantconferring mutations, and that the comprehensive measurements of resistance should be based on the sum of these interactions. It is possible that the $\operatorname{ramRA}$ haplotype in the highly resistant strain faced antagonistic mutations, whereas these mutations were absent in the complemented parental strain.

To gain an insight into the adaptive response of $S$. Enteritidis to high concentrations of ciprofloxacin, we determined the expression patterns of a wide range of genes in the susceptible, resistant, and highly resistant groups of $S$. Enteritidis. Most notably, the expression of genes, encoding the acrAB-tolC efflux pump and its activator $\operatorname{ram} A$, were significantly upregulated in almost all the strains of the resistant and highly resistant groups. In contrast, the gene, encoding porin ompF, was significantly downregulated in each strain of the resistant and highly resistant groups. Several studies that investigated ompF expression in FQs-resistant Salmonella illustrated the unclear role of this porin in contribution to FQs resistance (Giraud et al., 2000; O'Regan et al., 2009). Our data shown that the downregulation of $o m p F$ is a common adaptive response of $S$. Enteritidis, while the downregulation of ompC was less profound and more strain-dependent, suggesting its minor role in the development of Salmonella FQs resistance. It has been shown 


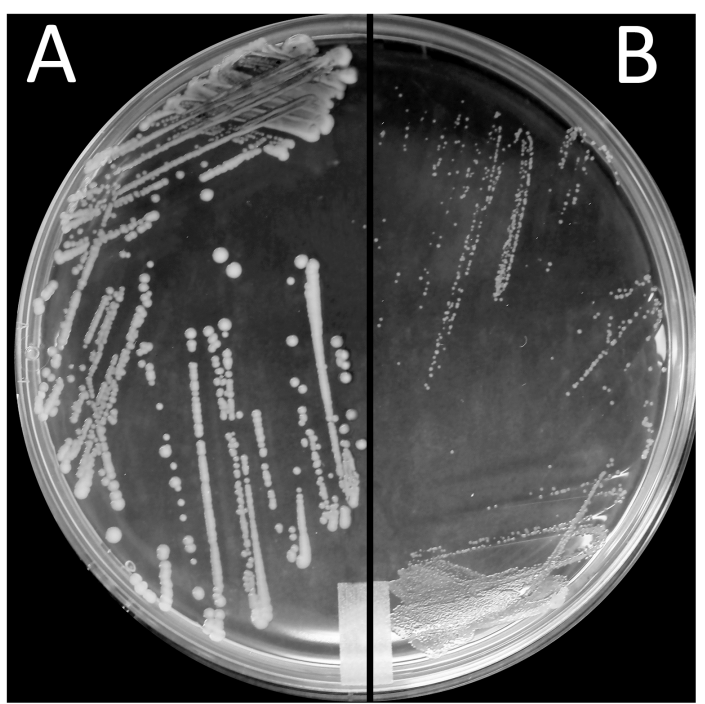

FIGURE 3 | Small colony variant phenotype. Morphological appearance of $S$ Enteritidis colonies on LB agar after incubation for $24 \mathrm{~h}$ at $37^{\circ} \mathrm{C}$. Shown are colonies of (A) parental (FQs susceptible) A-21 strain and (B) small colony variants (SCV) of A-21 spontaneous (FQs highly resistant) mutant strain.

that mutations in the marR regulator result in both an increase in $a c r B$ expression and a decrease in $\operatorname{ompF}$ expression (Randall and Woodward, 2002; Hooper and Jacoby, 2015). Our recent study showed that the rpoE sigma factor also inhibits the synthesis of outer membrane porins during the antimicrobial treatment of Escherichia coli O157 with a polycationic agent (Vidovic et al., 2018), further illustrating that the porin-mediated permeability plays a role in the development of resistance to various antimicrobial agents.

Comparing the expression of the genes and amino acid substitutions, the A-5 resistant strain showed a distinct characteristic compared to the rest of resistant mutants. Only the A-5 strain, once exposed to the subinhibitory concentration of ciprofloxacin did not up regulate the expression of the $\operatorname{ram} A$ gene. It is noteworthy to mention that the A-5 resistant strain acquired three amino acid substitutions (S83Y, D87G in GyrA and S80R in ParC), whereas other resistant mutants acquired one amino acid substitution at 83 position of GyrA. It has been documented that the number of QRDR amino acid substitutions has a detrimental effect on the stress adaptive physiology of several bacterial species, including Staphylococcus aureus (Horváth et al., 2012), Klebsiella pneumoniae (Tóth et al., 2014), and Escherichia coli (Johnson et al., 2015). Bacterial strains that acquire multiple QRDR mutations develop highlevel resistance against FQs and preserve fitness (Fuzi et al., 2017). In contrast, strains with fewer QRDR mutations must relay on the FQs non-specific mechanisms, primarily on efflux, an energetically demanding mechanism, which has a direct influence on bacterial fitness (Fuzi, 2016). To determine the relationship between the energetically favorable QRDR mutations and bacterial fitness, we performed the growth assays using the A5 resistant (multiple QRDR mutations) and its parental A-5 susceptible strain as well as the A-21 and A-33 resistant (single QRDR mutation) and their parental strains. Our data showed that the A-5 resistant strain has a similar growth curve as its parental strain, whereas the A-21 and A-33 resistant strains showed altered growth compared to its parental strain. Overall, this study provides evidence that $S$. Enteritidis with multiple QRDR mutations do not activate efflux to reach resistance to ciprofloxacin $(4 \mu \mathrm{g} / \mathrm{mL}$ ), while a strain with fewer QRDR mutations must activate efflux to reach the same MIC. Further, this stress adaptive response has an important effect on the overall fitness of $S$. Enteritidis.

Finally, this study revealed the existence of a small colony of variants (SCVs) phenotype among the resistant $S$. Enteritidis strains. These highly resistant strains of $S$. Enteritidis are characterized by a significant growth deficiency and small

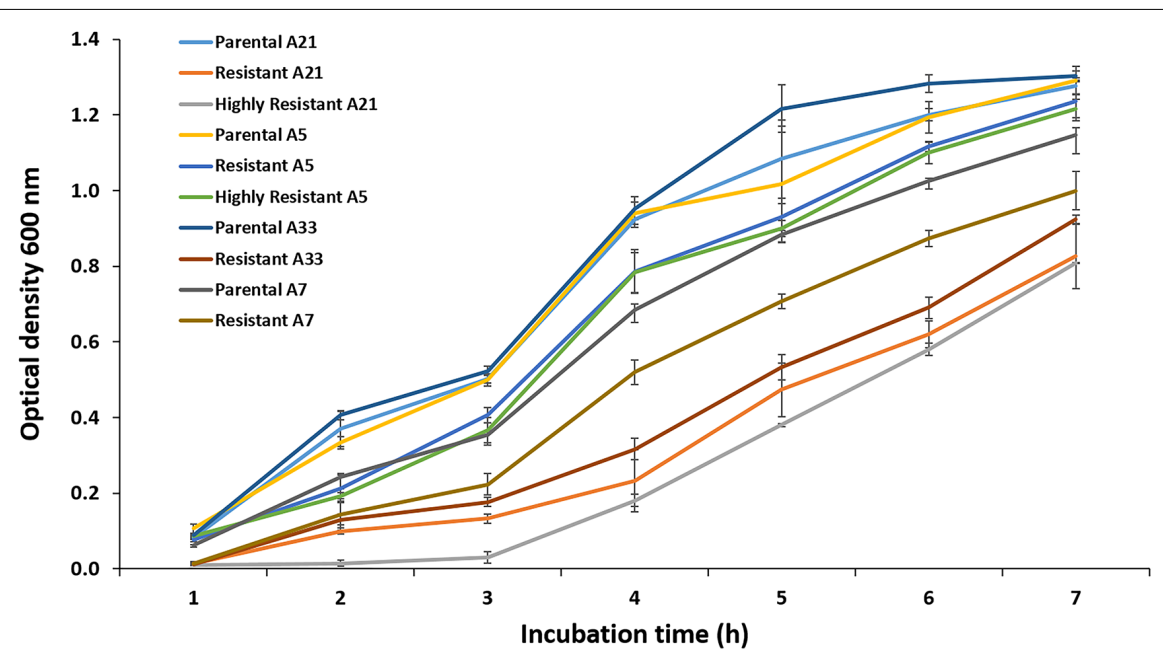

FIGURE 4 | Growth assay. The growth kinetics for the A-5, A-7, A-21, and A-33 parental and their resistant mutants were measured during the exponential growth phase. Also the growth curves were measured for the A-5 and A-21 highly resistant strains. The data correspond to the mean values of three biological replications. 
colony size compared to their parental strains. It has been demonstrated that the growth rate of an organism directly correlates to the antimicrobial resistance (Fuentes-Hernandez et al., 2015; Knopp and Andersson, 2018), with the more slowly replicating organisms being more resistant compared to their faster replicating counterparts. O'Regan et al. (2010) showed that in the absence of selective pressure, a spontaneous ciprofloxacin resistant $S$. Enteritidis strain after 20 passages reverted to its parental phenotype (e.g., reversal of all fitness cost except motility). They also showed that with increased fitness of the reverted strain ciprofloxacin susceptibility of this strain significantly increased. This physiological adaptation (i.e., growth rate) most likely represents another mechanism employed by $S$. Enteritidis to confer an extremely high level of resistance to FQs. It is interesting that only the A-5 mutants did not acquire SCV phenotype and showed altered growth compared to their parental strain. These strains preserved their original fitness while developing the high level of FQ resistance further indicating most likely the existence of unique compensatory mutations that maintain the high level of FQ resistance while maintaining the original strain fitness.

In summary, the development of FQs resistance among a population of clinical isolates of Salmonella spp. is of a great health concern. This study provides two important novel findings. The first, the deletions in the $\operatorname{ramRA}$ region, affecting the RamR binding site, and the amino acid substitution at position 87 of the GyrA are mutations significantly associated with the highly resistant $S$. Enteritidis strains, clearly illustrating their importance in conferring a high level of FQs resistance. The second, the deletion in the intergenic region of the $\operatorname{ram} R A$ operon provides resistance and reduced susceptibility

\section{REFERENCES}

Abouzeed, Y. M., Baucheron, S., and Cloeckaert, A. (2008). ramR mutations involved in efflux-mediated multidrug resistance in Salmonella enterica serovar Typhimurium. Antimicrob. Agents Chemother. 52, 2428-2434. doi: 10.1128/ AAC.00084-08

Bangtrakulnonth, A., Pornreongwong, S., Pulsrikarn, C., Sawanpanyalert, P., Hendriksen, R. S., Wang, D. L. F., et al. (2004). Salmonella serovars from humans and other sources in Thailand, 1993-2002. Emerg. Infect. Dis. 10, 131-136. doi: 10.3201/eid1001.02-0781

Baucheron, S., Coste, F., Canepa, S., Maurel, M. C., Giraud, E., Culard, F., et al (2011). Binding of the RamR repressor to wild-type and mutated promoters of the ramA gene involved in efflux-mediated multidrug resistance in Salmonella enterica serovar Typhimurium. Antimicrob. Agents Chemother. 56, 942-948. doi: 10.1128/AAC.05444-11

Baucheron, S., Tyler, S., Boyd, D., Mulvey, M. R., Chaslus-Dancla, E., and Cloeckaert, A. (2004). AcrAB-TolC directs efflux-mediated multidrug resistance in Salmonella enterica serovar Typhimurium DT104. Antimicrob. Agents Chemother. 48, 3729-3735. doi: 10.1128/AAC.48.10.37293735.2004

Blair, J. M. A., Bavro, V. N., Ricci, V., Modi, N., Cacciotto, P., Kleinekathofer, U., et al. (2015). AcrB drug-binding pocket substitution confers clinically relevant resistance and altered substrate specificity. Proc. Natl. Acad. Sci. U.S.A. 112, 3511-3516. doi: 10.1073/pnas.1419939112

Clinical and Laboratory Standards Institute [CLSI] (2015a). Methods for Dilution Antimicrobial Susceptibility Tests for Bacteria That Grow Aerobically: Approved Standard-Tenth Edition M07-A10. Villanova: National Committee for Clinical Laboratory Standards. to several antibiotics, including, ceftiofur, amoxicillin/clavulanic acid, azithromycin and tetracycline. Collectively, our study suggests complex interactions between mutations, bacterial adaptations, and FQs resistance.

\section{AUTHOR CONTRIBUTIONS}

SV conceived the study, carried out the experiments, and drafted the manuscript. RA carried out the experiments. AR performed the statistical analysis related to the gene expressions.

\section{FUNDING}

This project was supported by the Signature Program AES GAR grant.

\section{ACKNOWLEDGMENTS}

The authors gratefully acknowledge the technical support from Daniela Vidovic. This project was supported by the Signature Program AES GAR grant.

\section{SUPPLEMENTARY MATERIAL}

The Supplementary Material for this article can be found online at: https://www.frontiersin.org/articles/10.3389/fmicb. 2019.00729/full\#supplementary-material

Clinical and Laboratory Standards Institute [CLSI] (2015b). Performance Standards for Antimicrobial Susceptibility Testing; Twenty-Fifth Informational Supplement. Villanova: National Committee for Clinical Laboratory Standards.

Fuentes-Hernandez, A., Plucain, J., Gori, F., Pena-Miller, R., Reding, C., Jansen, G., et al. (2015). Using a sequential regime to eliminate bacteria at sublethal antibiotic dosages. PLoS Biol. 13:e1002104. doi: 10.1371/journal.pbio.1002104

Fuzi, M. (2016). Dissimilar fitness associated with resistance to fluroquinolones influences clonal dynamics of various multiresistant bacteria. Front. Microbiol. 7:1017. doi: 10.3389/fmicb.2016.01017

Fuzi, M., Szabo, D., and Csercsik, R. (2017). Double-serine fluroquinolone resistance mutations advance major international clones and lineages of various multi-drug resistant bacteria. Front. Microbiol. 8:2261. doi: 10.3389/fmicb.2017. 02261

Giraud, E., Cloeckaert, A., Kerboeuf, D., and Chaslus-Dancla, E. (2000). Evidence for active efflux as the primary mechanism of resistance to ciprofloxacin in Salmonella enterica serovar Typhimurium. Antimicrob. Agents Chemother. 44, 1223-1228. doi: 10.1128/AAC.44.5.1223-1228.2000

Gordon, M. A. (2008). Salmonella infections in immunocompromised adults. J. Infect. 39, 626-631. doi: 10.1016/j.jinf.2008.03.012

Graham, S. M. (2010). Nontyphoidal salmonellosis in Africa. Curr. Opin. Infect. Dis. 23, 409-411. doi: 10.1097/QCO.0b013e32833dd25d

Hartl, D. L. (2014). What can we learn from fitness landscapes? Curr. Opin. Microbiol. 21, 51-57. doi: 10.1016/j.mib.2014.08.001

Hooper, D. C., and Jacoby, G. A. (2015). Mechanisms of drug resistance: quinolone resistance. Ann. N. Y. Acad. Sci. 1354, 12-31. doi: 10.1111/nyas. 12830

Horváth, A., Dobay, O., Kardos, S., Ghidán, Á, Tóth, Á, Pászti, J., et al. (2012). Varying fitness cost associated with resistance to fluoroquinolones governs 
clonal dynamic of methicillin-resistant Staphylococcus aureus. Eur. J. Clin. Microbiol. Infect. Dis. 31, 2029-2036. doi: 10.1007/s10096-011-1536-z

Johnson, J. R., Johnston, B., Kuskowski, M. A., Sokurenko, E. V., and Tchesnokova, V. (2015). Intensity and mechanisms of fluoroquinolone resistance within the $\mathrm{H} 30$ and $\mathrm{H} 30 \mathrm{Rx}$ subclones of Escherichia coli sequence type 131 compared with other fluroquinolone-resistant E. coli. Antimicrob. Agents Chemother. 59, 4471-4480. doi: 10.1128/AAC.00673-15

Kearse, M., Moir, R., Wilson, A., Stones-Havas, S., Cheung, M., Sturrock, S., et al. (2012). Geneious basic: an integrated and extendable desktop software platform for the organization and analysis of sequence data. Bioinformatics 28, 1647-1649. doi: 10.1093/bioinformatics/bts199

Knopp, M., and Andersson, I. D. (2018). Predictable phenotypes of antibiotic resistance mutations. mBio 9:e770-18. doi: 10.1128/mBio.00770-18

Larkin, M. A., Blackshields, G., Brown, N. P., Chenna, R., McGettigan, P. A., McWilliam, H., et al. (2007). Clustal W and Clustal $\mathrm{X}$ version 2.0. Bioinformatics 23, 2947-2948. doi: 10.1093/bioinformatics/btm404

Martinez-Martinez, L., Pascual, A., and Jacoby, G. A. (1998). Quinolone resistance from a transferable plasmid. Lancet 14, 797-799. doi: 10.1016/S0140-6736(97) 07322-4

Nikaido, E., Yamaguchi, A., and Nishino, K. (2008). AcrAB multidrug efflux pump regulation in Salmonella enterica serovar Typhimurium by RamA in response to environmental signals. J. Biol. Chem. 283, 24245-24253. doi: 10.1074/jbc. M804544200

Okoro, C. K., Kingsley, R. A., Connor, T. R., Harris, S. R., Parry, C. M., AlMashhadani, M. N., et al. (2012). Intra-continental spread of human invasive Salmonella Typhimurium pathovariants in sub-Saharan Africa. Nat. Genetics 44, 1215-1221. doi: 10.1038/ng.2423

O’Regan, E., Quinn, T., Frye, J. G., Pages, J. M., Porwollik, S., Fedorka-Cray, P. J., et al. (2010). Fitness cost and stability of a high-level ciprofloxacin resistance phenotype in Salmonella enterica serotype Enteritidis: reduced infectivity associated with decreased expression of Salmonella pathogenicity island 1 genes. Antimicrob. Agents Chemother. 54, 367-374. doi: 10.1128/AAC. 00801-09

O’Regan, E., Quinn, T., Pages, J. M., McCusker, M., Piddock, L., and Fanning, S. (2009). Multiple regulatory pathways associated with highlevel ciprofloxacin and multidrug resistance in Salmonella enterica serovar Enteritidis: involvement of ramA and other global regulators. Antimicrob. Agents Chemother. 53, 1080-1087. doi: 10.1128/AAC. 01005-08

Pers, C., Sogaard, P., and Pallesen, L. (1996). Selection of multiple resistance in Salmonella Enteritidis during treatment with ciprofloxacin. Scand. J. Infect. Dis. 28, 529-531. doi: 10.3109/00365549609037954

Piddock, L. J., Ricci, V., McLaren, I., and Griggs, D. J. (1998). Role of mutation in the $g y r A$ and $\operatorname{par} C$ genes of nalidixic-acid-resistant salmonella serotypes isolated from animals in the United Kingdom. J. Antimicrob. Chemother. 41, 635-641. doi: $10.1093 / \mathrm{jac} / 41.6 .635$
Randall, L. P., and Woodward, M. J. (2002). The multiple antibiotic resistance (mar) locus and its significance. Res. Vet. Sci. 72, 87-93. doi: 10.1053/rvsc.2001. 0537

Reddy, E. A., Shaw, A. V., and Crump, J. A. (2010). Community-acquired bloodstream infections in Africa: a systematic review and meta-analysis. Lancet Infect. Dis. 10, 417-432. doi: 10.1016/S1473-3099(10)70072-4

Redgrave, L. S., Sutton, S. B., Webber, M. A., and Piddock, L. J. V. (2014). Fluoroquinolone resistance: mechanisms, impact on bacteria, and role in evolutionary success. Trends Microbiol. 22, 438-445. doi: 10.1016/j.tim.2014. 04.007

Ricci, V., and Piddock, L. J. V. (2009). Ciprofloxacin selects for multidrug resistance in Salmonella enterica serovar Typhimurium mediated by at least two different pathways. J. Antimicrob. Chemother. 63, 909-916. doi: 10.1093/jac/dkp054

Rupali, P., Abraham, O. C., Jesudason, M. V., John, T. J., Zachariah, A., Sivaram, S., et al. (2004). Treatment failure in typhoid fever with ciprofloxacin susceptible Salmonella enterica serotype Typhi. Diagnostic Microbiol. Infect. Dis. 49, 1-3. doi: 10.1016/j.diagmicrobio.2003.12.002

Scallan, E., Hoekstra, R. M., Angulo, F. J., Tauxe, R. V., Widdowson, M. A., Roy, S. L., et al. (2011). Foodborne illness acquired in the United States-major pathogens. Emerg. Infect. Dis. 17, 7-15. doi: 10.3201/eid1701.P11101

Schmittgen, T. D., and Livak, K. J. (2008). Analyzing real-time PCR data by the comparative CT method. Nat. Protocols 3, 1101-1108. doi: 10.1038/nprot. 2008.73

Tamura, K., Dudley, J., Nei, M., and Kumar, S. (2007). MEGA4: molecular evolutionary genetics analysis (MEGA) software version 4.0. Mol. Biol. Evol. 24, 1596-1599. doi: 10.1093/molbev/msm092

Tóth, Á, Kocsis, B., Damjanova, I., Kristóf, K., Jánvári, L., Pászti, J., et al. (2014). Fitness cost associated with resistance to fluroqunolones is diverse across clones of Klebsiella pneumoniae and may select for CTX-M-15 type extendedspectrum B-lactamase. Eur. J. Clin. Microbiol. Infect. Dis. 33, 837-843. doi: 10.1007/s10096-013-2022-6

Vidovic, S., Medihala, P., Dynes, J. J., Daida, P., Vujanovic, V., Hitchcock, A. P., et al. (2018). Importance of the RpoE regulon in maintaining the lipid bilayer during antimicrobial treatment with the polycationic agent, chlorhexidine. Proteomics 18, 1-9. doi: 10.1002/pmic.201700285

Conflict of Interest Statement: The authors declare that the research was conducted in the absence of any commercial or financial relationships that could be construed as a potential conflict of interest.

Copyright $(02019$ Vidovic, An and Rendahl. This is an open-access article distributed under the terms of the Creative Commons Attribution License (CC BY). The use, distribution or reproduction in other forums is permitted, provided the original author(s) and the copyright owner(s) are credited and that the original publication in this journal is cited, in accordance with accepted academic practice. No use, distribution or reproduction is permitted which does not comply with these terms. 